\title{
Las experiencias pedagógicas de los educadores de Inzá, apuesta por una educación del campo
}

\author{
THE PEDAGOGICAL EXPERIENCES OF INZÁ EDUCATORS: BETTING ON FIELD EDUCATION \\ I AS EXPERIÊNCIAS PEDAGÓGICAS DOS PROFESSORES DE INZÁ, A APOSTA PARA A EDUCAÇÃO DO CAMPO
}

\author{
María Isabel González Terreros* \\ Sonia Rodríguez Salcedo** \\ Katherine Viasús Poveda***
}

\begin{abstract}
Resumen
El presente artículo es producto del trabajo investigativo y pedagógico realizado en el municipio de Inzá, Cauca, en el marco de la práctica pedagógica comunitaria de la Licenciatura en Educación Comunitaria de la Universidad Pedagógica Nacional. La intención es reconocer y comprender las apuestas y experiencias pedagógicas que realizan los educadores de Inzá, junto con sus comunidades. Estas experiencias, que parten de la escuela, se insertan en los territorios, en las familias, en las organizaciones, para generar alternativas frente a problemáticas que los educadores vieron en sus territorios; por esta razón condensan algunos elementos de la educación del campo.
\end{abstract}

\section{Abstract}

This article is a product of research and pedagogical work accomplished in the municipality of Inzá, Cauca, in the context of the teaching internships of the Community Education Program from Universidad Pedagógica Nacional. The goal is to recognize and understand the pedagogical proposals and experiences of educators from Inzá and their communities. These experiences, which originate from the schools, are applied to the territories, families and organizations to generate alternatives to the problems faced by the teachers in their territories, which cause them to condense some elements of rural education.

\section{Resumo}

O presente artigo é produto da pesquisa pedagógica desenvolvida no município de Inzá, Cauca, no marco da prática pedagógica comunitária da Licenciatura em Educação Comunitária da Universidade Pedagógica Nacional. A intenção é reconhecer e compreender as apostas e experiências pedagógicas dos educadores de Inzá junto com suas comunidades. Estas experiências, nascidas na escola, são inseridas nos territórios, nas famílias e nas organizações, para gerar alternativas frente às problemáticas evidenciadas pelos professores nos seus territórios. Por essa razão, alguns elementos da educação rural foram condensados.

\section{Palabras clave}

experiencias pedagógicas, narrativas docentes, educación del campo, ruralidad, subjetividad

\section{Keywords}

pedagogical experiences; teacher narratives; field education; rurality; subjectivity

\section{Palavras chave}

experiências pedagógicas; narrativas docentes; educação do campo; ruralidade; subjetividade

Fecha de recepción: 12 de julio de 2016 / Fecha de aprobación: 08 de septiembre de 2016

* Docente e investigadora de la Universidad Pedagógica Nacional de Colombia (UPN). Correo electrónico: migonzalez@pedagogica.edu.co

** Licenciada en Educación Comunitaria con énfasis en Derechos Humanos (UPN). Correo electrónico: sonrsalcedo10@gmail.com

*** Licenciada en Educación Comunitaria con énfasis en Derechos Humanos (UPN). Correo electrónico: katherineviasus16@gmail.com 


\section{Educación del campo}

La investigación que presentamos es el resultado de las reflexiones y los aportes de una práctica pedagógica comunitaria (PPC) que se realizó desde el programa de Educación Comunitaria de la Universidad Pedagógica Nacional con los maestros rurales del municipio de InzáCauca quienes han desarrollado propuestas pedagógicas que contienen elementos de la educación del campo.

Esta práctica se realizó entre 2014 y 2015 en el municipio de Inzá, un territorio con múltiples riquezas socioculturales y arqueológicas, compartido por población indígena y campesina que se posiciona desde su identidad cultural, política y social que se evidencia en los procesos organizativos que se lideran en las comunidades. Es un territorio comunitario en búsqueda de autonomía, en el cual convergen diferentes poblaciones que tienen su identidad, saberes y costumbres fortalecidas, es un espacio de construcción colectiva en el cual se han configurado horizontes de sentido transformadores como apuesta a fomentar proyectos de vida digna.

En este contexto se han desarrollado algunas propuestas educativas que son lideradas por educadores de la región que forman parte de las organizaciones locales y que, a la vez, están afiliados a Asoinca, el sindicato de maestros más antiguo del país y más importante de la región. Precisamente, en el marco del trabajo de la subdirectiva sindical de Inzá se propuso un ejercicio de formación pedagógica con los maestros de las escuelas. De manera conjunta -la subdirectiva de Inzá, los maestros de la zona, y docentes y estudiantes de la Universidad-, planteamos un ejercicio con la intención de que los maestros visibilizaran las propuestas pedagógicas que han venido adelantando por años en sus instituciones y cuya importancia e impacto para las escuelas y comunidades no han sido suficientemente reconocidas.

La idea fue que, a partir de ese reconocimiento, los maestros reflexionaran acerca de su propuesta pedagógica, la potenciaran y actualizaran. Como parte de este ejercicio, ellos estuvieron acompañados en un proceso que permitió la escritura de las narrativas pedagógicas de sus experiencias, con las cuales participaron en el I Encuentro de Experiencias Pedagógicas Alternativas, realizado en el municipio.
De ese proceso, encontramos que las experiencias pedagógicas de los maestros tienen un fuerte componente territorial. Es decir, reconocen elementos de su contexto y los vinculan a la escuela como parte de los procesos de enseñanza de los educandos y para potenciar los procesos sociales que se realizan en las comunidades. Existe un vínculo entre la escuela y la comunidad a partir de la comprensión de su territorio, de las personas que lo habitan, de los recursos que poseen, de las situaciones problemáticas que se presentan, elementos claves para el ejercicio docente y el trabajo conjunto con la comunidad.

Se trata, por ejemplo, de proyectos educativos en lectoescritura en los cuales los estudiantes leen con sus familiares y generan reflexiones; proyectos acerca de producción agrícola que sirven para alimentar a los jóvenes de las escuelas; proyectos territoriales que pretenden el reconocimiento del lugar, y el fortalecimiento y actualización de la identidad.

Estas iniciativas dejan ver que los educadores del municipio están potenciando una educación del campo, propuesta de educación latinoamericana que toma distancia de la educación rural. La educación del campo le apuesta a la

[...] formación de sujetos colectivos, sujetos que luchan por formar parte de la dinámica social, para ser construidos como sujetos políticos, capaces de influir en la agenda política [... que] da paso a la emergencia de nuevos educadores que se preguntan por la pedagogía, la sociedad, educadores constructores de políticas, pensadores de la pedagogía y sujetos de prácticas sociales'. (Salete, 2009, p. 15).

La apuesta por la formación colectiva y comunitaria, el reconocimiento del lugar que las personas ocupan en el mundo, los proyectos emancipatorios para transformar la realidad en que se vive, la apuesta por un proyecto de país, la conciencia de que existe la posibilidad de cambiar, el reconocimiento de la política en la educación, la lucha por la tierra y el territorio, son elementos claves de la educación del campo que piensa la educación para la incidencia colectiva de los

1 Traducción del portugués al español por las autoras. 
trabajadores del campo, a diferencia de la educación rural que plantea una educación del individuo para articularse al mundo del mercado.

La educación del campo busca incidir en su realidad a partir del reconocimiento del territorio como espacio donde sus actores y dinámicas producen conocimiento, de manera que se otorgue un lugar a las organizaciones sociales, a los sujetos que trabajan la tierra y por el territorio. Busca, además posicionar la lucha social y los proyectos del campo que tienen apuestas frente a un proyecto de país, que se configuran en alternativas político-pedagógicas para un proyecto de sociedad que se construye colectivamente.

Desde esta perspectiva, la educación piensa la escuela de manera distinta a la oficial, no se trata de la formación individual del estudiante para que se inserte lo mejor posible al mundo del mercado, por el contrario, considera:

[...] la escuela como el vínculo hacia las comunidades, hacía una humanización más plena, la desalienación del trabajo en la tierra y a favor de la democratización, participación política en la defensa y afirmación de la organización colectiva, de manera que se fortalezca el poder popular y la formación de militancia para las organizaciones. Desde aquí, se asume el proceso pedagógico como proceso político que se vincula orgánicamente con la organización social de las comunidades en pro de la justicia social, la democracia radical y los valores humanistas. (Salete, 2005, p. 161).

En algunas experiencias pedagógicas de los maestros de Inzá, se asume una apuesta colectiva y transformativa desde su quehacer pedagógico, se trata de un ideal político de transformación de la realidad, en donde se opta por re-significar la construcción de sujetos que trabajan y viven para el beneficio conjunto de sus comunidades. Frente a ello mencionan los educadores con los que consolidamos nuestro trabajo de investigación,

La educación en la vida rural debe permitir reconocer las poblaciones que la habitan, sus valores, sistemas de representación y narrativas, configurar un sentido de pertenencia, un nosotros [...] Debe ofrecer posibilidades para integrar $y$ fortalecer procesos colectivos que sean fuente de conocimiento en la escuela y en las comunidades. (Comité de Educación de la ACIT, 2011, p. 20).
Los educadores tienen apuestas pedagógicas en las cuales realizan proyectos que vinculan el trabajo de sus estudiantes con el territorio, con las organizaciones, con las familias; iniciativas potenciadoras de una educación del campo que están configurando, tal vez no con un discurso que lo sustente, pero sí desde una práctica que muestran procesos educativos que se piensan sus escenarios desde lo social, cultural, económico y político en aras de fortalecer sus territorios y apostar por un país distinto.

\section{Reconocimiento de experiencias pedagógicas desde la investigación acción}

En el trabajo con los maestros de Inzá partimos de reconocer las necesidades de los educadores y de su contexto, antes que llegar con propuestas que no dialogan con sus experiencias y se convierten en discurso que en poco fortalecen los procesos educativos. Este criterio nos llevó conocer las propuestas pedagógicas de docentes que día tras día, lejos de la ciudad, metidos tierra adentro, construyen escenarios educativos contextualizados al territorio. De allí que nos planteamos un trabajo investigativo para la comprensión de las relaciones socioeducativas de la región.

Para ello, retomamos los planteamientos de la investigación acción (IA), como un enfoque pertinente en los procesos de construcción de conocimiento a partir de las lecturas del contexto, del reconocimiento de los sujetos como agentes sociocríticos de la realidad. La IA le otorga un lugar central al educador, quien al comprender sus prácticas educativas puede potenciar su quehacer constantemente, pues es sujeto activo que indaga y problematiza su propia práctica pedagógica, como lo afirman Jhon Elliott (2000), Wilfred Carr (1996), entre otros.

La investigación acción constituye una opción metodológica que permite la expansión del conocimiento y da respuestas concretas a problemáticas que se van planteando los participantes de la investigación, que a su vez se convierten en coinvestigadores que participan activamente en todo el proceso que se origina, producto de reflexiones constantes (Elliott, 2000, p. 32)

Este enfoque de investigación no ve la realidad como dada, sino dándose y por ello permite indagar acerca de las problemáticas del contexto desde las percepciones que tienen los sujetos, por medio del diálogo y la 
autoreflexión sobre las situaciones que los aquejan. Para este caso, la pertinencia de documentar las experiencias pedagógicas de los educadores inzaeños, nace de la necesidad de reflexionar sobre su quehacer y proyección educativa. Ello permitió a los docentes crear espacios de reflexión sobre las iniciativas pedagógicas que habían desarrollado, y a la vez lograr compresiones sobre la educación para la vida, como lo afirma una profesora,

No solo se trata de asistir a la escuela para aprender contenidos, estos deben responder a la vida colectiva del campo, en la que nos relacionamos con el medio, con las plantas, con el trabajo agrícola, con la huerta, con la lluvia y las fases de la luna -esto es vital para el campo-, y debe ser parte del proceso de enseñanza [...] una pedagogía que integre a los distintos actores y sectores del territorio en función de intereses genuinamente colectivos. (Comité de Educación de la ACIT, 2011, p. 22).

Lo importante de la labor de los educadores, es el contacto que se puede establecer no solo con los estudiantes, sino con las familias y comunidades, por ello se vuelve una profesión humana, humanizante, de ahí la importancia del compromiso que se tenga con el lugar donde se habita, por quienes comparten y porque la lucha es conjunta, es beneficio para todos. (Morales, 2015).

Estas comprensiones de la escuela y lo pedagógico trascienden las barreras oficiales de la institución educativa y se insertan en la vida misma de las comunidades, donde los maestros forjan prácticas que permiten el reconocimiento de los demás seres humanos y de la colectividad. En este sentido, la IA nos permitió reconocer y valorar el trabajo cotidiano y diario de los maestros del municipio, y desde este reconocimiento propusimos fortalecer las comprensiones del quehacer educativo de los educadores inzaeños. Así se construyó una ruta de trabajo que se elaboró colectivamente y que tuvo diferentes momentos como reseñaremos a continuación.

\section{Reconociendo las experiencias pedagógicas}

Teniendo en cuenta los intereses de algunos profesores del municipio que forman parte de Asoinca, nos acercamos a las instituciones educativas con la intención de conocer las experiencias pedagógicas que aquellos han venido implementando.

En este primer momento visitamos las instituciones educativas por días, en cada escuela convocamos a los educadores para socializar la propuesta y para que participaran en el taller acerca de las narrativas docentes en las prácticas educativas rurales, insumo principal para el I Encuentro de Experiencias Pedagógica-Alternativas del Municipio de Inzá- Cauca. La idea fue que, a partir del reconocimiento de sus proyectos alternativos, los docentes eligieran cuáles querían documentar según la pertinencia e importancia para su institución. Así, un grupo de educadores o de manera individual se responsabilizaron de realizar la narración escritural de la experiencia pedagógica.

De las 16 instituciones educativas que conforman el municipio, contamos con la voluntad y apoyo de 24 educadores de 10 instituciones. En las visitas a estas últimas, pudimos conocer algunas experiencias que agrupamos en los siguientes ejes:

- Lectoescritura: cuyo énfasis se centró en que, por medio de la lectura y escritura crítica de textos, los estudiantes se apropiaran de elementos que reconocen en su contexto y les permiten asumir un lugar frente a este.

- Territorialidad: los proyectos desarrollados en este eje se encaminan a comprender lo físico, lo social y lo cultural, permitiendo a los educadores y estudiantes consolidar una estrecha relación entre la comunidad, el territorio y los saberes que confluyen allí

- Relación de la escuela y la comunidad rural: aquí, las experiencias aluden al trabajo colectivo, la responsabilidad social, la cooperación, la solidaridad y la orientación del trabajo con la comunidad rural.

\section{Acompañando el proceso escritural de las experiencias}

Para esta parte del proceso se plantearon unos criterios que orientaron la escritura de las experiencias pedagógicas por medio de las narrativas docentes. La escritura se abordó de forma narrativa, comprendiéndola 
como una herramienta metodológica, pedagógica y de comunicación que permite volver sobre la experiencia significativa que realizan los educadores en su labor, no solo para generar la posibilidad de reconocerse y valorar su práctica pedagógica, sino como un ejercicio formativo en el que los docentes reflexionan y escriben sobre el qué, cómo, para qué y qué sucedió en su práctica pedagógica, siendo protagonistas de su propia historia. Para ello, se trabajó en tres momentos:

- Punto de partida de la experiencia: los educadores dieron a conocer cómo surgió la experiencia, las necesidades que llevaron a la consolidación de la propuesta, el por qué de la misma, con el objetivo de comprender los elementos que justifican las iniciativas. Por otra parte, consideramos importante dar cuenta de la intencionalidad política y pedagógica: qué quisieron lograr, para qué y a quiénes fue dirigida la iniciativa. Para ello, se realizó colectivamente la construcción de los avances escriturales en forma narrativa, con el fin de contemplar los ejercicios escriturales como un escenario de reflexión y de expresión de las cotidianidades de los educadores.

- Desarrollo de la experiencia: los educadores reflexionaron a partir de las preguntas: ¿Cómo se desarrolló la experiencia?, ¿quiénes forman o formaron parte del proceso?, ¿cuál fue el impacto que se generó, las herramientas utilizadas y la ruta metodológica? Así, a lo largo del relato consideramos pertinente evidenciar de forma transversal lo significativo y lo considerado alternativo por ellos, en cada una de las experiencias.

- Valoración de las experiencias: los educadores realizaron un balance del proceso bajo las siguientes preguntas orientadoras: ¿Qué es fundamental y qué se debe cambiar en el desarrollo de las experiencias?, ¿qué se necesita mejorar y cómo potenciar la experiencia? A su vez, indagamos por el sentido, la trascendencia, y las reconfiguraciones y replanteamientos que han surgido al volver sobre las experiencias, para desde allí, hilar las conclusiones con la escritura de un artículo como reflexión final del proceso escritural, que fue publicado en una revista realizada para tal fin, como lo presentaremos más adelante.

\section{Realizando el I Encuentro de Experiencias} Pedagógicas

Posterior a la escritura de las experiencias por parte de los educadores, se llevó a cabo el I Encuentro de Experiencias Pedagógicas del Municipio de Inzá, que estuvo acompañado con la edición de la revista Educación y pedagogía rural desde las prácticas docentes.

El evento tuvo como objetivo visibilizar, fomentar y articular las experiencias pedagógicas y proyectos de educación considerados alternativos, en el municipio. En él participaron aproximadamente 350 educadores, estudiantes y administrativos de las instituciones educativas, padres y madres de familia, así como miembros de la comunidad en general. El encuentro fue posible gracias a los educadores, quienes presentaron sus prácticas pedagógicas que nutren la educación del campo; se contó, además, con la asistencia y participación de: la Administración Municipal de Inzá; la Dirección de Núcleo Estudiantil; Asoinca; la Subdirectiva Municipal de Docentes de Inzá, con quienes se trabajó de manera mancomunada para la resignificación de las prácticas y el papel de los educadores.

En este evento se realizó el lanzamiento de la revista Educación y Pedagogía Rural, en la cual se publicaron los artículos de los educadores teniendo en cuenta las siguientes orientaciones: aportes de las experiencias pedagógicas a la resignificación de la educación rural; relación entre las experiencias pedagógicas con la comunidad que habita el territorio; reflexión crítica que dejan las experiencias a los educadores.

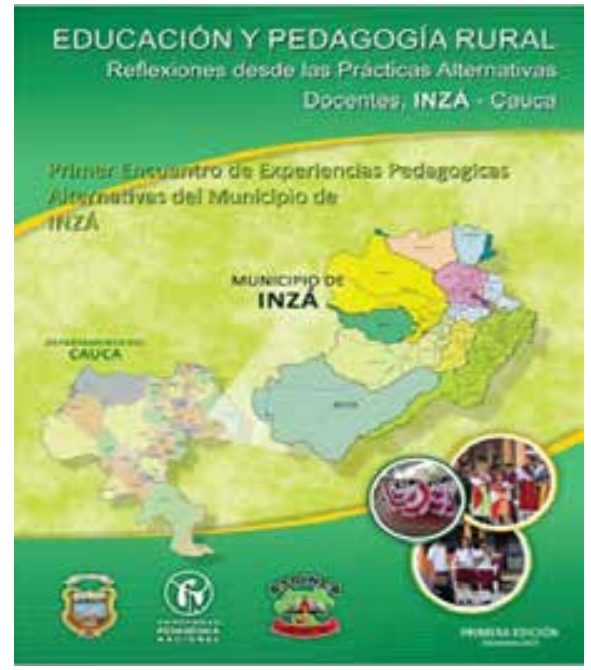

Figura 1. Portada primera edición de la Revista Educación y Pedagogía Rural, 2014 
Cada institución participante se unió a la idea de realizar una feria de experiencias pedagógico-alternativas. Igualmente, presentó un stand para que todos los educadores asistentes e invitados pudiesen conocer de primera mano el trabajo a nivel pedagógico que se realiza en el municipio, se exhibieron los diferentes trabajos y resultados de los proyectos alternativos teniendo en cuenta los ejes temáticos.

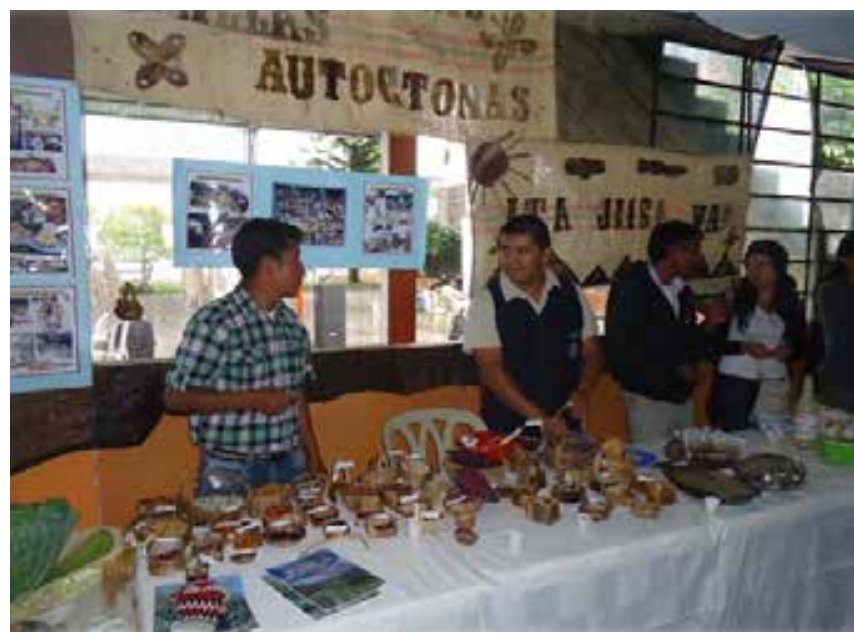

Figura 2. Stand Institución Educativa Santa Rosa Jiisa Yat, octubre 2014

\section{Reflexionando sobre la educación del campo}

Al retomar las experiencias, los escritos sobre estas, y en diálogo constante con los docentes y la comunidad, seguimos reflexionado acerca de la potencia de las experiencias pedagógicas en contextos rurales. Se trataba de una educación que iba más allá de la educación rural que propone el campo al servicio de la ciudad; por el contrario las experiencias trascendían la apuesta de la formación escolar y se vinculaba a los procesos de sus comunidades. Este acercamiento nos llevó a recocer elementos de la educación en el campo en las experiencias pedagógicas del municipio.

Comprendemos que la educación del campo representa dinámicas, prácticas y discursos propios que se contraponen a las formas de concebir la educación como transmisión de conocimientos absolutos dentro de un determinado lugar o espacio. Esta invita a pensar el quehacer de la educación por fuera de lógicas modernizantes que parecieran ser la bandera de cambios y transformaciones desde lineamientos estatales; propone pensar en conocimientos que lleven a pedagogías coherentes con las dinámicas sociales del campo, es decir, orientarlas a las necesidades propias y demandas del contexto rural insertos en un mundo global.

Así pues, entender el ejercicio educativo como un quehacer para la vida, es quizá el mayor de los intereses de los educadores inzaeños, ya que han optado por construir desde la escuela y fuera de ella, herramientas y comprensiones que los estudiantes puedan asumir no únicamente en los cuadernos de clase, sino en la vida de cada uno, en sus pensamientos y sus sueños. Por ejemplo, conversar con ellos sobre qué es ser campesino o indígena, escuchar las historias de sus antepasados, las iniciativas que han gestado como comunidad, discutir sobre las necesidades que se tienen allí y sobre el papel de cada persona en la comunidad y las organizaciones, son algunas de las reflexiones constates de los educadores y de las cuales hemos aprendido con ellos. Allí existe un conocimiento importante en lo pedagógico que vincula la escuela a su contexto sociocultural, y se convierte en un sentido de vida colectivo. En un taller, una educadora mencionó,

Para nosotros el interés no es que ellos se formen para que vayan a la capital a ser mano de obra, nuestro interés como educadores es que ellos se eduquen, que aprendan y enseñen a ser personas que aporten con ideas, sueños y acciones a su propia comunidad. (Ermelisa 2015, Conversatorio-taller Educación del Campo. Inzá-Cauca).

La escuela que está dentro de la comunidad propende porque los jóvenes y niños comprendan su contexto local y global para que asuman otras formas de sentirse y de estar en el mundo, para que se comprendan como sujetos de agenciamiento de su vida. La escuela se considera como un lugar de encuentro, no solo de conocimientos, sino también como un lugar que forma parte del entramado comunitario y contribuye a la formación de sujetos colectivos.

\section{Narrativas y subjetividad docente: reflexiones sobre la educación del campo}

El proceso de investigación acción en el que junto con los educadores reflexionamos y visibilizamos las experiencias pedagógicas, nos permitió ver el papel 
del educador como un sujeto en movimiento que le apuesta a propuestas alternativas en educación desde su práctica docente. Para mostrar ello, a continuación, presentamos, en una primera parte, las construcciones narrativas de los docentes y, en la siguiente, el papel de ellos como sujetos ético políticos.

\section{Narrativas docentes: desde las experiencias pedagógicas}

Aquí evidenciamos las experiencias pedagógicas teniendo en cuenta el ejercicio de narrativas docentes que elaboramos conjuntamente durante el proceso investigativo. La experiencia, como lo afirma Larrosa (1995), conforma un dispositivo pedagógico por medio del cual podemos ver distintos momentos por los que pasa la reflexión de sí y de las experiencias. La experiencia, para este autor, es "eso que me pasa. No eso que pasa, sino eso que me pasa" (2009, p. 14), es decir se trata de una pregunta del sujeto sobre sí, sobre su vida y la forma como aquello que nos pasa, nos configura.

Algunos de los docentes que participaron de este trabajo están vinculados con organizaciones sociales que se configuran en un proyecto de vida colectivo, ello hace que la experiencia docente sea más enriquecida y profunda. Los sujetos, cuando están organizados, tienden a articular su profesión con los sentidos de la organización que, en el caso de Inzá, están relacionados con la lucha territorial y cultural; en ese sentido, los docentes piensan la escuela con vínculos en el territorio, proponen proyectos que afecten el contexto social y, por ello, consideramos que sus apuestas están vinculadas con la educación del campo.

Para dar cuenta de las narrativas docentes, elegimos algunas que agrupamos en los tres ejes de trabajo mencionados arriba: relación escuela/comunidad rural, lectoescritura y territorialidad.

\section{Eje relación escuela/comunidad rural}

La relación de la escuela con la comunidad rural se refiere al "quehacer docente hacia afuera de la escuela". Es decir, hacia el contexto y cotidianidad de los estudiantes, de sus grupos familiares y organizaciones sociales. Pues se considera la importancia de entender los espacios formativos permeados de conocimientos y saberes propios a sus habitantes, gestando propuestas que fortalezcan su identidad, su expresión corporal, su personalidad, sus habilidades y potencialidades en pro de construir un tejido social que convoque a la población en general. Aquí hacemos referencia a la narrativa docente denominada "Escuela de Danza- Biblioteca Casa del Pueblo, en Guanacas".

Esta surge en la vereda Guanacas en 2007, bajo la iniciativa de algunas educadoras que a su vez se consideran madres, campesinas y habitantes del municipio; estos roles les plantean la necesidad de trabajar con los niños de la vereda con el fin de potenciar sus capacidades artísticas orientadas hacia el fortalecimiento de su identidad. Más que ensayar los bailes característicos de la región, la experiencia busca fortalecer el sentido de pertenencia y arraigo, en este caso, campesino, logrando que los niños y jóvenes conozcan sus saberes, costumbres y raíces. Frente a la intencionalidad de la experiencia, expresa una educadora,

La comunidad es vinculada a este proceso por medio de concursos, eventos, bazares y fiestas, en los que además de visibilizar las capacidades artísticas y corporales de los niños, se lleva un mensaje de unidad y de fortalecimiento de lo comunitario y su identidad, apoyando iniciativas comunales que tengan que ver con lo formativo, lo organizativo y sociocultural (Narrativas Docentes-Escuela de Danza Biblioteca Casa del Pueblo, 2014).

El elemento imperativo encontrado en este proceso de investigación ha sido el vínculo directo que los educadores tienen con propuestas organizativas que desde hace varios años han trabajado por la consolidación de iniciativas que les otorgue un lugar y unas condiciones particulares como habitantes del campo. Estos vínculos se han fortalecido a través de una perspectiva comunitaria y comunal de entender su quehacer: "Educar para la vida del campo" ha sido una afirmación constante en los educadores y padres de familia, quienes tienen la posibilidad de desdibujar las fronteras de la escuela para consolidar aportes desde y hacia la comunidad. 
Considerando, pues, las iniciativas educativas como parte fundamental de un proyecto comunitario de trasformación social en la región, el ejercicio pedagógico de educar se gesta como un escenario de resistencia colectiva y comunitaria que convoca a quienes forman parte de la comunidad como educadores, estudiantes y padres de familia a ser partícipes de los escenarios de construcción no solo de la escuela y lo que tiene que ver con ella, sino de las iniciativas que se dan por fuera de ella como escenarios de posibilidad para tejer vínculos colectivos y comunitarios.

\section{Eje lectoescritura}

En diferentes análisis que los educadores han hecho de las problemáticas de su contexto escolar, se han dado cuenta que existen dificultades en procesos de lectura y escritura. Desde los educadores se comprenden los procesos lectoescriturales como estrategia interdisciplinar, cuya base es el afecto hacia la lectura y la escritura, para luego abordar aspectos sobre comprensión e interpretación de textos, y contextos que potencien las habilidades del pensamiento, el lenguaje, la comunicación y creación del mundo que los rodea. Ejemplo de las experiencias pedagógica son: Leer es mi cuento y Proyecto Lector Institución Educativa Santa Teresa.

Leer es mi cuento, de la Institución Educativa empresarial San José, surge de la poca motivación de los estudiantes por la lectura y escritura, el bajo nivel de comprensión, interpretación y la dificultad para expresar las ideas. Los estudiantes manifestaron que las prácticas de lectura y escritura no han existido como hábito en su cotidianidad, ni en el interior de la escuela; así como tampoco se han adecuado espacios que favorezcan e incentiven el gusto por leer y escribir. Por eso, se involucró a las familias en la experiencia,

A través de los procesos escriturales de los niños quisimos vincular a las familias, por eso se propuso la lectura colectiva en familia de un libro, para que después los niños contaran la experiencia de compartir este espacio con ellos. De igual manera, la escritura permitió que se hiciera un ejercicio familiar, pues en la mayoría de los casos, los niños tomaban como referencia sus familias para construir historias (Narrativas Docentes-Institución Educativa San José, 2014).
La lectura y escritura dentro de esta experiencia dejó de ser un ejercicio de obligación en la clase, pues los padres y la comunidad comenzaron a participar y apoyar los procesos. Así, se convirtió en un espacio en el que los estudiantes tienen la posibilidad de expresar sus intereses, su experiencia cotidiana, mediante el recurso de la imaginación para crear y recrear los textos y los escritos.

Uno de los espacios más bonitos, era cuando se convocaba a la comunidad para reconocer los ejercicios creativos de nuestros estudiantes, ellos sentían valorado su trabajo y las familias manifestaban la importancia de hacer parte de los procesos de aprendizaje de sus hijos. (Narrativas Docentes-Institución Educativa San José, 2014).

Por otra parte, en el Proyecto Lector Institución Educativa Santa Teresa, los docentes veían a sus estudiantes como reproductores y trascriptores de documentos; de allí surge la idea de fortalecer la capacidad de leer y escribir de manera crítica y reflexiva desde sus vivencias y relacionarlas con la cotidianidad de la institución y la comunidad. Se idearon estrategias agradables dentro y fuera del aula, en las cuales los estudiantes fueran capaces de expresar y exponer sus ideas y empezaran a tener un pensamiento autónomo y crítico.

Es necesario decir que estos chicos y chicas se merecen una oportunidad para ser mejores seres humanos y que somos los maestros los que tenemos la oportunidad de ayudarlos y que por estar en la profesión más bonita de todas las profesiones, nos asiste el deber sagrado de hacer todo lo que esté a nuestro alcance para que nuestros estudiantes se eduquen y formen en un clima de amistad, respeto, ayuda mutua, tolerancia y ante todo mucho amor. (Narrativas DocentesInstitución Educativa Santa Teresa, 2014).

La construcción de espacios lúdicos dentro y fuera del salón de clase fue crenado hábitos de lectoescriturales agradables y educativos junto con los padres de familia, para mejoramiento de las relaciones interpersonales. Lecturas de manera alegre y divertida de obras literarias sencillas y adecuadas a la edad de los estudiantes y de su contexto fue una de las estrategias que contribuyó a mejorar los niveles de lectura. Además, se propuso la escritura libre de cuentos explorando su creatividad, se realizaron coplas musicales alusivas a las lecturas referidas, elaboración de pinturas y dibujos de los cuentos. Ello generó 
que los estudiantes comprendieran la importancia de relacionar las experiencias cotidianas con los ejercicios académicos de la lectura y la escritura.

\section{Eje territorialidad}

La pertinencia de las experiencias educativas en un contexto rural debe propiciar escenarios de encuentro en donde los estudiantes puedan tejer vínculos con el territorio como espacio en el que confluyen sus saberes, sus cosmovisiones, sus costumbres y demás aspectos que los caracterizan.

Comprendemos que la territorialidad identifica a los sujetos como campesinos e indígenas dentro un espacio o lugar en donde converge lo físico, lo social y lo cultural. Esto es lo que permite consolidar una estrecha relación con el territorio pensando en iniciativas que desde la escuela propendan por gestar alternativas de soberanía alimentaria, territorial y el aprovechamiento de los recursos naturales desde un enfoque sociocultural y comunitario. Ello se muestra con las experiencias "Recuperación de las semillas nativas, de la Institución Educativa Jiisa Yat, en Santa Rosa; La voz de la naturaleza, mediante la recuperación y repoblamiento de especies de flora y fauna, Institución Educativa A' Kwe Uus Yat (Casa de las Estrellas), en el Lago- Inzá Cauca", que además de trabajar por el aprovechamiento de los recursos naturales para beneficio colectivo, difunde en los estudiantes, iniciativas sobre el cuidado del lugar en que se habita.

La experiencia "Recuperación de las semillas nativas" ha venido trabajando en la recuperación de semillas y preparación de diferentes platos típicos, vinculando a estudiantes, docentes, padres de familia y comunidad en general. Esta iniciativa se da con el fin de generar una propuesta de alimentación sana y orgánica para las familias y los estudiantes de la vereda, porque se identificó que los alimentos que, en general, se consumen se traen de la cabecera municipal o ciudades aledañas, representando la compra y reproducción de productos tratados genéticamente por países industrializados. Al respecto, comenta un educador:

La escuela en la ruralidad, tiene que ser mucho más dada hacia afuera, hacia el campo, hacia el compartir y el sentir de las personas que nos están rodeando, debe ser más proyectada hacia la comunidad. Por eso la importancia que se le debe dar a la Asociación de padres de familia, a las juntas de acción como una forma de fomentar la participación. Por ello debe vincularse a los padres a las actividades comunitarias que tenga que ver con lo educativo, para que no se pierda el sentido social de la educación. (Institución Educativa Jissa Yat, 2014).

Desde esta experiencia se optó como alternativa comunitaria, la recolección y siembra de semillas nativas como una actividad escolar, con la que se difundiría la conciencia de aprovechamiento de recursos, la creación de huertas caseras, y la utilización de terrenos aledaños a la institución para la reproducción de semillas y alimentos que la comunidad soberanamente pueda consumir. En este sentido, el objetivo de la experiencia se fundamenta en implementar el banco de semillas propias para el fortalecimiento de la autonomía alimentaria de la institución y comunidad en general.

Esta iniciativa se trabaja a nivel institucional como proyecto educativo comunitario (PEC), en donde los mayores (adultos mayores del resguardo, sabedores tradicionales), tienen el papel de contextualizar a los estudiantes frente a los usos, nombres, aspectos de su cosmovisión relacionados con las semillas y demás recursos.

Por otra parte la experiencia "La voz de la naturaleza, mediante la recuperación y repoblamiento de especies de flora y fauna" ha trabajado en la construcción de un sendero ecológico en el que se puedan sembrar las semillas nativas que se han logrado recuperar en la vereda por parte de estudiantes y núcleos familiares, así como la siembra de árboles nativos con el fin de proteger y construir nacimientos de agua, y la incorporación de huertas escolares que puedan servir para la alimentación de los estudiantes y profesores de la institución educativa. Es una experiencia institucional, en la que cada grado trabaja en dos horas diarias en la recolección de materiales para la siembra, construcción y mantenimiento del sendero ecológico. De igual manera en la recolección y abastecimiento de alimentos para la tienda escolar, cuyos ingresos se utilizan para la compra de materiales e insumos necesarios en el sendero. Aquí, se manifiesta la apuesta por defender la soberanía que tienen en el lugar 
en el que habitan, y la necesidad de recuperar no solo sus costumbres y saberes, sino también de recuperación de su hábitat y medio ambiente.

Consideramos que este acompañamiento pedagógico es un aporte a la resignificación del quehacer pedagógico de los educadores, a la comprensión sus prácticas a partir de los procesos escriturales, como escenarios de crítica y reflexión politizada que permite cuestionarse acerca de las alternativas posibles de conocimiento que se pueden generar, afirmando que lo educativo y pedagógico debe aportar a repensar la sociedad.

\section{Escenarios de reflexión: una mirada a la subjetividad docente}

Las experiencias pedagógicas evidencian un horizonte en el que los maestros piensan su profesión más allá de la institución, de los desarrollos cognitivos y se insertan a reflexionar su territorio, su comunidad y su papel como educadores en contextos rurales que forman parte de una sociedad.

La importancia recuperar la cotidianidad de la labor de los educadores y situarla como conocimiento desde un posicionamiento ético-político es fundamental en la medida en que le permite al educador valorar su saber y su hacer que ha estado construyendo, pero que no había sido reconocido. De manera que el ejercicio narrativo tocó la subjetividad del educador, porque le posibilitó visibilizar sus experiencias pedagógicas, el conocimiento que ha construido a partir de ellas, y a la vez le generó reflexiones para seguir avanzando y fortaleciendo sus apuestas político pedagógicas.

Algunos maestros consideran la importancia de sus experiencias dentro y fuera de la escuela, porque se convierten en una voz de aliento y motivación para sus pares.

Lo importante es entonces mostrar desde las narrativas a nuestros colegas que a pesar de las dificultades esta es una profesión muy bonita, Ilena de compromiso, amor [...] es una lucha día a día, pero una lucha compartida, por eso si hacemos una reflexión otros deben conocerla, llevar un mensaje motivador. (Liz, Conversatoriotaller Educación del Campo, 2015).
Los educadores, cuando reflexionan desde su lectura de realidad, hacen a su vez una lectura de sí mismos en esa realidad. Es decir, vuelven sobre las dimensiones que los han configurado como sujetos, permitiéndoles identificar los procesos culturales, políticos y sociales que han compuesto la experiencia y su lugar en el mundo. Se trata de un proceso de volver y reflexionar sobre su historia para comprender sus horizontes de sentido y pensar de otro modo. Apoyándonos en Zemelman (2010), "para leer la realidad debemos saber leer el movimiento constituyente de los sujetos, lo que tropieza con diferentes obstáculos en general asociados a formas de pensar que rehúyen simplemente en lo que carece de formas claramente definidas" (p. 357).

La lectura crítica de la realidad configura la subjetividad de los educadores comprendiendo la apropiación de su lugar como sujeto social y lucha por el reconocimiento de los sujetos en los contextos que los constituyen. Por tal razón, el proceso de la lectura crítica en los procesos pedagógicos conduce a los educadores a verse, pensarse, relacionarse, juzgarse y comprometerse, porque este ejercicio construye nuevas formas de comprensión y reconstrucción de conocimientos.

Desde los lugares de significación que consolidan los sujetos se configuran modos distintos de leer la realidad, de abrirse a lo desconocido, de desnaturalizar las visiones de futuro centradas en lógicas individuales. Desde allí, la resistencia de los sujetos pasa por la conformación de un tejido social, que logra colectivamente construir una identidad con los otros, rescatando su voz y su lugar, reformulando y construyendo nuevas relaciones con la realidad.

Quisimos acercarnos a la constitución y consolidación de una subjetividad emancipadora que recupere el papel de los sujetos como constructores de realidad, que se ocupan de gestar acciones distintas en sus contextos, y de configurar otro tipo de relaciones.

La subjetividad emancipadora, como hemos querido mencionar, cobra un papel relevante en la consolidación de escenarios colectivos, de acciones conjuntas, pues desde allí se plantea recuperar una perspectiva de vida social, que tenga en cuenta la proliferación de luchas que viene dotando de protagonismo a los sujetos, que pese a un orden dominante han logrado 
construir alternativas frente a lo que antes se consideraba imposible. Estas luchas, Ilevadas a cabo desde diferentes lugares sociales y políticos han apostado a la reconfiguración de la realidad, nutriendo reivindicaciones de diversos sectores que han tejido vínculos diferentes que expresan su potencial transformador.

Como educadores relacionamos los proyectos tanto comunitarios del municipio como los educativos de las instituciones, así también estamos educando, porque cuestiono lo que estoy enseñado, cómo lo evalúo y cómo desde otros escenarios puedo vincular los saberes que nos ofrece el territorio, para que esto sea pertinente con las verdaderas necesidades que hay en la región.

En ese sentido, desde estas iniciativas se apuesta por la resignificación y valoración de conocimientos propios o locales, cuestionando el tipo de conocimiento que pretende mostrarse como universal y único; allí los saberes que emergen desde su contexto significan el punto central para proponer alternativas de transformación social, de igual manera ubicando el contexto rural como un referente de construcción identitaria ligada a la organización sociopolítica.

\section{Conclusiones}

Las propuestas educativas de los maestros dejan ver que se trata de una apuesta que fortalece las prácticas de vida de las comunidades y los procesos organizativos, de allí que retomemos la Educación del Campo como una iniciativa política y pedagógica que se gesta de la mano de las poblaciones rurales, con el fin de reconocer y afirmar la realidad cultural, saberes, identidades, memorias e historias que constituyen las necesidades y proyectos de futuro que colectivamente se generan en los territorios.

Aunque los educadores de Inzá hacen mención a la educación rural, consideramos que ésta se enmarca más en la educación del campo, porque se ajusta a su práctica pedagógica social y política. Precisamente, los educadores le apuestan a propuestas colectivas de construir comunidad a través de la educación, de pensar el quehacer de los educadores y los educandos como un proceso de resistencia desde las poblaciones, por construir prácticas pedagógicas pertinentes con su realidad. Así, consideramos oportuna la definición conjunta que el Colectivo Diatriba construye frente a las apuestas educativas ligadas a la movilización social, “una construcción colectiva basada en descubrir y compartir saberes en un proceso de enseñanza que supere los intereses individuales" (Colectivo Diatriba, 2013, p. 18).

Consideramos que estos elementos se vinculan a los procesos pedagógicos en el territorio como una apuesta que involucra a los jóvenes, niños y adultos en la lucha por el establecimiento de relaciones de territorialidad que implica una relación humana de reconocimiento con el otro.

Por otra parte, en términos formativos, el ejercicio de los maestros sobre la escritura de las experiencias pedagógicas, según manifestaron, fue una forma de reflexionar en la manera que se estaban expresando y juzgando sus experiencias y su práctica educativa, lo que llevó a incentivarlos para continuar con los procesos pedagógicos y escriturales.

En este sentido, no solo se trataba de volver sobre sus prácticas pedagógicas, sino de potenciarse a nivel profesional, personal y colectivo. Por ello, consideramos la pertinencia y el incentivo que generó ver condensadas sus reflexiones en la revista, porque nos expresaban que ver materializado, valorado y no evaluado por estándares de calidad su trabajo, los hacía volver a pensar en la posibilidad de los cambios en la educación en el campo.

\section{Referencias}

Aguilera, A., González, M. y Torres, A. (2015). Reinventando la comunidad y la política: formación de subjetividades, sentidos de comunidad y alternativas políticas en procesos organizativos locales. Bogotá: Universidad Pedagógica Nacional.

Arias, L. (2014). Narrativas Docentes-Escuela de Danza, Biblioteca Casa del Pueblo. Inzá, Cauca.

Carr, W. (1996) Una teoría para la educación. Hacia una investigación educativa critica. España: Editorial Morata

Colectivo Diatriba. (2013). Educación y Movimientos Sociales en América Latina. Chile: Editorial Quimantú.

Comité de Educación de la ACIT (2011). Proyecto Educativo Rural Intercultural PERI.

Elliott, J. (2000). El cambio educativo desde la investigación-acción. Madrid: Editorial Morata.

González, M. y Aguilera, A. (2013). Ni profesor, ni maestro... Educador, la figura emblemática en los procesos de educación alternativa de América Latina. Revista Pedagogía y Saberes, 39,77-86 Bogotá. Universidad Pedagógica Nacional.

Institución Educativa San José (2014). Narrativas Docentes-Leer es mi Cuento. Cauca.

Institución Educativa Santa Rosa Jiisa Yat (2014). Narrativas Docentes-Recuperación de Semillas Nativas. Cauca. 
Larrosa, J. (1995). Tecnologías del yo educación. Notas sobre la construcción y la mediación pedagógica de la experiencia de sí. En: J. Larrosa. Escuela poder y subjetivación. Madrid: Ediciones de la Piqueta.

Larrosa, J. (2009). Experiencia y alteridad en educación. En: C. Skliar y J. Larrosa (comp.) Experiencia y alteridad en educación. Rosario, Argentina: Flacso-Homo Sapiens.
Morales, M. (2015). Conversatorio- taller Educación del Campo Inzá. Documento de trabajo.

Salete, C. (2005). Principios da educação no MST. En: I.T. Iterra. Caderno de educação. 13a. ed. São Paulo: Editora Expressão Popular.

Zemelman, H. (2010). Sujeto y subjetividad, la problemática de las alternativas como construcción posible. Polis, 9(27), 355-366.

\section{Diálogo del conocimiento}

Este artículo es resultado de la práctica pedagógica comunitaria de estudiantes del Programa de Educación Comunitaria de la Universidad Pedagógica Nacional, y representa un interés particular por el quehacer del educador en el contexto de la educación del campo, logrando un interesante ejercicio de articulación con los maestros rurales pertenecientes a la Asociación de Institutores y Trabajadores de la Educación del Cauca (Asoinca).

Los resultados de este proceso dejan entrever el lugar ético-político de las experiencias pedagógicas de los educadores de Inzá (Cauca), pues se constituyeron reflexiones fundamentadas en procesos escriturales de sus propias narrativas pedagógicas y puestas en diálogo con otros maestros.

Para lograr un trabajo que surgiera desde la lectura del contexto y del reconocimiento de los sujetos políticos, en este caso los maestros de Inzá, se optó por los planteamientos de la investigación acción (IA), como enfoque investigativo que logra la reflexión y la construcción de conocimiento, en este caso desde la práctica pedagógica de los maestros, puesta en escena desde sus propias reflexiones. Es decir, la narrativa se consolida en el elemento central, desde el cual los maestros logran enunciar esos elementos constitutivos de su experiencia.

Dentro de las experiencias de los maestros se resalta la permanente relación con el contexto, con la comunidad, la identidad y algunos procesos que fortalecen la formación de los estudiantes. Toda esta apuesta política de los maestros se fundamenta en la educación del campo, como aquella que logra dar un sentido distinto a la educación, en donde se busca formar a las colectividades como sujetos políticos, que logran proyectar la pervivencia de las comunidades. Se menciona en diferentes apartados, que esta categoría propuesta como educación del campo, se distingue de la educación rural, por ser esta una determinación del Estado, por el contrario, la educación del campo surge desde las comunidades y logra tener un sentido de la formación para la vida.

La educación del campo desde esta perspectiva es la que da el sentido del ser maestro en el contexto rural, ya que son múltiples los elementos que deben ser comprendidos e integrados para realizar un proceso que trasciende a la enseñanza y el aprendizaje. A la vez, es el maestro quien llena de sentido a la educación de campo, cuando decide que esta es una apuesta política pertinente para la formación de las comunidades.

Considero de gran importancia el proceso escritural desarrollado, tanto por parte de los maestros quienes lograron sistematizar sus propias experiencias, pero también de las escritoras de este artículo, ya que parten de sistematizar su propio ejercicio de práctica investigativa. Es una excelente forma de construcción de conocimiento que permite la constitución y el fortalecimiento de otras experiencias pedagógicas.

Angie Benavides 